\title{
Prevalencia del consumo de drogas en pacientes atendidos en urgencias de adultos
}

\author{
Sofía Piñero-De Fuentes, M. Sc., ${ }^{(1)}$ Evelyn Medina-0 rozco, Farmacéutica, ${ }^{(1)}$ \\ Maritza Rojas, M. Sc.(2)
}

\begin{abstract}
Piñero-De Fuentes S, Medina-Orozco E, Rojas M. Prevalencia del consumo de drogas en pacientes atendidos en urgencias de adultos. Salud Publica Mex 1998;40:234-240.
\end{abstract}

\section{Resumen}

Objetivo. Conocer la asociación entre consumo de marihuana, co caína y/o bazuco, y causas de ingreso de pacientes al Hospital Central de Valencia,Venezuela. Material y métodos Se estudiaron 148 individuos recibidos en el área de urgencias, debido a lesiones por agresión (LA), accidentes de tránsito (AT), accidentes de trabajo (ATB), lesión autoinfringida (LAU) e intoxicaciones (IN T). Se aplicó un cuestionario y un análisis toxicológicos. Resultados. Se encontró un consumo de drogas, solas y/0 asociadas, de $23.6 \%$ (13.5\% cocaína, $7.4 \%$ marihuana y $2.7 \%$ ambas). De los pacientes positivos para cocaína, $50 \%$ ingresaron por $L A ; 20 \%$ por IN T; $10 \%$ por LAU; $5 \%$ por ATB, y $15 \%$ por otras causas. De los positivos para marihuana, $36.4 \%$ ingresaron por AT; $27.3 \%$ por ATB; $18 \%$ por $\mathrm{LA} ; 9.09 \%$ por IN T, y $9.09 \%$ por otros motivos. Los ingresos por LA fueron de $75 \%$, y por ATB, de $25 \%$, para drogas en combinación. El consumo alcohólico asociado a cocána fue de 50\%; a marihuana, de 9\%; a combinación, de $25 \%$. Conclusiones Estos hallazgos demuestran que uno de cada cuatro ingresos por causa traumática está asociado al abuso de drogas, solas 0 en combinación, lo que revela una estrecha relación causaefecto.

Palabras clave: abuso de sustancias; alcoholismo; accidentes de trabajo; accidentes de tránsito; heridas y lesiones; Venezuela

\author{
Piñero-De Fuentes S, Medina-Orozco E, Rojas M. \\ Drug consumption prevalence \\ in adult patients attending the emergency room. \\ Salud Publica Mex 1998;40:234-240.
}

\begin{abstract}
A bstract
Objective To determine the association between patient admission due to trauma and the consumption of marihuana, cocaine and bazooka (basic cocaine paste) at the Central Hospital of Valencia, Venezuela. Material and methods 148 subjects were studied who had entered the emergency room due to lesions caused by aggression (AL), traffic accidents (TA), work-related accidents (W RA), selfinflicted injury (SII) and intoxication (IN T).A questionnaire was applied and toxicology analyses performed. Results D rug consumption, alone or in combination, was found in $23.6 \%$ of individuals $(13.5 \%$ cocaine, $7.4 \%$ marihuana and $2.7 \%$ both). $O f$ the cocaine positive, $50 \%$ entered for $\mathrm{AL}, 20 \%$ for INT, $10 \%$ for SII, $5 \%$ for W RA and $15 \%$ for other causes. of the marihuana positive, $36.4 \%$ entered for TA, followed by $27.3 \%$ for W RA, $18.18 \%$ for AL, $9.09 \%$ for IN T and $9.09 \%$ for other causes. For combined drug consumption, entries for $\mathrm{AL}$ were $75 \%$ and for W RA, $25 \%$. Alcohol consumption associated to cocaine was $50 \%$, to marihuana, $9 \%$ and to combined drugs, $25 \%$. Conclusions. These results show that one of every four hospital admissions for trauma is associated to drug abuse, alone or in combination. This reveals a close cause-effect relationship between the use of psychoactive drugs and trauma.
\end{abstract}

Key words: substance abuse; alcoholism; accidents, occupational; accidents, traffic; wounds and injuries; Venezuela

(1) Investigadora del Centro de Investigaciones Toxicológicas, Universidad de Carabobo (CITUC), Valencia, Venezuela.

(2) Directora del CITUC, Valencia, Venezuela. 
E 1 consumo de drogas en Venezuela ha aumentado progresivamente en los últimos años, y se ha ido relacionando cada vez más con las situaciones de violencia que se presentan y su consiguiente saldo de personas ingresadas a centros hospitalarios por hechos vinculados a este consumo. Mota G. ${ }^{1}$ realizó un estudio en Caracas, entre 50 individuos que ingresaron al servicio de urgencias del Hospital Dr. Leopoldo Manrique Tejera de esa ciudad, por heridas causadas con armas de fuego o blancas, de los cuales $81.6 \%$ consumían cocaína. Otros estudios realizados en el país reflejan que las drogas más consumidas son marihuana, cocaína y bazuco (pasta básica de coca). ${ }^{2, *}$

Según estudios previos, ${ }^{3}$ el consumo se inicia a partir de los 12 años, y aumenta entre los 16 y los 21 años. De los delitos relacionados con el consumo de drogas, los de carácter violento son más fácilmente cuantificados; la violencia misma es la causa más común de muertes. En investigaciones previas se ha demostrado que los delitos violentos derivados del consumo de cocaína y otras drogas son una de las causas de muerte de sus usuarios. ${ }^{4,5}$ Es conocido que los consumidores de drogas como alcohol, marihuana, inhalantes, anfetaminas y cocaína, cometen un número significativamente mayor de actos antisociales que los individuos que no las consumen. ${ }^{6}$

Existen diversos estudios que explican la correlación entre el hábito del consumo de drogas y la generación de hechos violentos. ${ }^{6-9}$ Entre otros, Spunt y colaboradores ${ }^{7}$ realizaron un estudio sobre el papel de la marihuana en homicidios en una correccional del estado de Nueva York, en los Estados Unidos de América (EUA). Los resultados revelaron que la marihuana fue la droga más utilizada por los encuestados y que en su gran mayoría la consumían 24 horas antes de cometer el homicidio. Es sabido que esa droga, además de tener efectos directos en la salud, está asociada con problemas tales como homicidios, suicidios, accidentes de tránsito, de trabajo, ausentismo laboral, delincuencia, etcétera. Hasta donde se sabe, en Venezuela no se han llevado a cabo investigaciones relacionadas con ingresos en los servicios de urgencias, que inclu-

\footnotetext{
* Bello LA, Figueroa M y Reyes M. Evaluación del abuso de sustancias psicoactivas en hospitales generales, centros de detención y centros de tratamiento para farmacodependientes. Caracas: Comisión Nacional Contra el Uso Ilícito de las Drogas, 1993. Documento no publicado.

Aponte J. Estudio sobre el consumo de drogas en estudiantes. Caracas: Fundación Venezuela Libre de Drogas, 1990. Documento no publicado.

Encuesta Nacional sobre el consumo de drogas lícitas e ilícitas en estudiantes de educación media. Caracas: Ministerio de la Familia/Fundación José Félix Ribas, 1990. Documento no publicado.
}

yan análisis toxicológicos para determinar la influencia del consumo de drogas en la generación de hechos violentos. En consideración a lo anterior, en este estudio se planteó determinar la prevalencia del consumo de drogas entre los pacientes atendidos por causas traumáticas en la sala de urgencias del Hospital Central de Valencia como representativa -por su dimensión e importancia- de los centros hospitalarios del país, tratando de establecer la correlación entre las variables estudiadas.

\section{Material y métodos}

La población estaba formada por 2630 pacientes que ingresaron, en el lapso de una semana (del 18 al 25 de julio de 1995), al área de urgencias del Hospital Central de Valencia, que es el más grande de la ciudad. La selección de la muestra estuvo condicionada a que el ingreso fuera por causa traumática, como lesión por agresión (LA), accidentes de tránsito (AT), accidentes de trabajo (ATB), lesión autoinfringida (LAU) e intoxicaciones (INT), y a que los pacientes se encontraran en estado consciente para la toma de la muestra de orina y la realización de la entrevista; de este modo se eligieron 148 sujetos $(5.63 \%)$ para el estudio.

Una vez identificado el paciente y previo su consentimiento escrito, se realizó la entrevista mediante cuestionario validado, ${ }^{10}$ para conocer antecedentes personales (sexo, edad, estado civil, nivel educativo, ocupación), estado de salud y hábito alcohólico; se le preguntó, además, si a la fecha del estudio, consumía marihuana, cocaína y/o bazuco, para determinar su potencial consumo de drogas. Dicho cuestionario es el utilizado por el Centro de Estudios sobre el Problema de Drogas, de la Universidad de Carabobo, Venezuela, en el programa Vigilancia Epidemiológica del Consumo de Drogas 1994. ${ }^{11}$ El consumo de alcohol, para el momento del ingreso, fue determinado a partir del interrogatorio y del examen físico, lo que describía estado de conciencia y presencia o ausencia de aliento alcohólico. No se determinó alcoholemia porque el trabajo se realizó en una población muy particular (pacientes traumatizados), y la toma de muestra de sangre fue considerada invasiva.

\section{A nálisis toxicológicos}

El personal encargado de aplicar la encuesta tomó una muestra de orina puntual a todos los individuos, para garantizar de esa forma la procedencia de la misma. Este equipo de personas fue debidamente entrenado y estuvo conformado por enfermeros y médicos recién graduados. 
Para los análisis toxicológicos se seleccionaron la cocaína (con su derivado bazuco) y la marihuana, debido a que son las de mayor consumo entre la población venezolana., ${ }^{2, *}$ El método empleado fue el inmunoenzimático (EMIT-DAU), ${ }_{12}^{12}$ el cual es sensible y específico para este tipo de análisis, ${ }^{13}$ de manera que la concentración de droga en la muestra puede medirse con base en la actividad enzimática. ${ }^{14}$ Para identificar la presencia de cocaína y bazuco se determinó benzoilecgonina, metabolito principal de estas drogas e indicador biológico de exposición a las mismas. Para detectar marihuana se determinó la presencia del metabolito principal del $\Delta^{9}$ Tetrahidrocannabinol (THC), el ácido 11-nor- $\Delta^{9}$ THC-9 carboxílico.

Las muestras de orina puntuales $(100 \mathrm{ml})$ fueron recolectadas en envases estériles y almacenadas a $-25^{\circ} \mathrm{C}$ sin tratamiento previo, para su posterior análisis. La cantidad de orina para la técnica utilizada fue de $50 \mu \mathrm{l}$, y cada muestra fue procesada por duplicado. En la calibración del instrumento se utilizaron igualmente patrones por duplicado y con un nivel de detección de $300 \mathrm{ng} / \mathrm{ml}$ para benzoilecgonina y $100 \mathrm{ng} / \mathrm{ml}$ para el 11-nor- $\Delta^{9}$-THC-9 carboxílico, valores recomendados por el National Institute on Drug Abuse de EUA, para el método inmunoenzimático. ${ }^{15}$

\section{Método estadístico}

Las observaciones, los datos de la entrevista y los resultados de las determinaciones analíticas, se resumieron en tablas y gráficos usando para su análisis frecuencias absolutas y relativas. La técnica para el análisis de la diferencia entre los porcentajes de respuestas afirmativas en relación con el total de sujetos investigados, según sexo, edad, causa de ingreso y tipo de droga consumida, fue chi cuadrada, a un nivel de significación de 5\% o menos. Se utilizó el programa estadístico SPSS versión 5.0 para Windows.

El tamaño de la muestra estuvo condicionado por factores como el estado consciente del paciente y el consentimiento del individuo para participar en el estudio.

\footnotetext{
* Bello LA, Figueroa My Reyes M. Evaluación del abuso de sustancias psicoactivas en hospitales generales, centros de detención y centros de tratamiento para farmacodependientes. Caracas: Comisión Nacional Contra el Uso Ilícito de las Drogas, 1993. Documento no publicado.

Aponte J. Estudio sobre el consumo de drogas en estudiantes. Caracas: Fundación Venezuela Libre de Drogas, 1990. Documento no publicado.

Encuesta Nacional sobre el consumo de drogas lícitas e ilícitas en estudiantes de educación media. Caracas: Ministerio de la Familia/Fundación José Félix Ribas, 1990. Documento no publicado.
}

\section{Resultados}

El número de pacientes evaluados fue de 148: 116 (78.4\%) fueron del sexo masculino. El rango de edades estuvo entre los 13 y los 60 años, y el promedio fue de 30 años.

Los datos revelan que el consumo de las drogas estudiadas, en forma independiente o asociadas, se hizo presente en $23.6 \%$ del total de casos estudiados (148); asimismo, el consumo de cocaína predominó en $57.14 \%$ del total de casos positivos (20/35). Al ser entrevistados los 148 pacientes, 22\% (33/148) refirieron ser consumidores de marihuana, cocaína y/o bazuco. Al relacionar estos datos con los del laboratorio se encontró que de los 33 sujetos consumidores, 22 (15\%) resultaron positivos a alguna de las drogas mencionadas, y de los 115 que manifestaron no ser consumidores, $13(9 \%)$ resultaron positivos a las pruebas de laboratorio. Del total de hombres y mujeres, 26.7 y $12.5 \%$, respectivamente, resultaron positivos al análisis de las drogas mencionadas, en general solas o combinadas. De los 35 pacientes con resultados positivos a las pruebas toxicológicas, 20 (13.5\%) fueron positivos para cocaína, $11(7.4 \%)$ para marihuana y $4(2.7 \%)$ para ambas drogas (cuadro I).

Los resultados del consumo de drogas por grupo de edad se indican en el cuadro II, donde se aprecia que en el rango de 18-30 años predominó el consumo de cocaína y/o bazuco en $55 \%$ del total de positivos para benzoilecgonina $(11 / 20)$, y el consumo de marihuana, en $54.5 \%$, porcentaje calculado igualmente con base en el total de positivos para esta droga (6/11).

En la figura 1 se muestra la distribución de la población según la causa de ingreso. Setenta y cuatro de los ingresos (50\%) se debieron a lesión por agresión causada con arma blanca, arma de fuego y objetos punzopenetrantes. La segunda causa de ingreso fueron los accidentes de tránsito $(16.9 \%, 25 / 148)$.

Entre los ingresos debidos a lesión por agresión se registró un consumo de cocaína, bazuco y/o marihuana de 20.3\% (15/74); en ingresos por accidente de tránsito, $16 \%$ (4/25) resultaron positivos para marihuana, y no se encontró un consumo de las otras drogas estudiadas. En los casos de intoxicación se pudo observar un consumo de $26.3 \%$ (5/19); en los accidentes de trabajo, de $33.3 \%(5 / 15)$, y en los casos por causa autoinfringida, de $40 \%(2 / 5)$ (cuadro III).

De los 148 casos estudiados 66 (44.6\%) eran solteros, y $48(32.4 \%)$, casados. En unión libre y divorciados había $28(18.9 \%)$ y $4(2.7 \%)$, respectivamente. Respecto a los 35 casos positivos al consumo de drogas, se registró que $9(25.7 \%)$ eran casados; 21 (60\%), 


\section{Cuadro I}

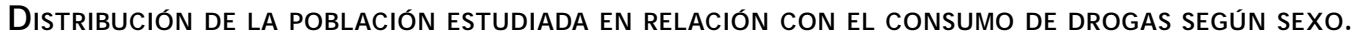 Hospital Central de Valencia, Venezuela, 1995}

M etabolito

Masculinos ( $n=116) \quad$ Femeninos $(n=32) \quad$ Frecuencias absolutas $(n=148) \quad$ Frecuencias relativas $\%(f=100)$

\begin{tabular}{lrrrr} 
Benzoilecgonina & 19 & 1 & 20 & 13.5 \\
\hline Ac.11-nor- $\Delta^{9}$-thc-9 carboxílico & 9 & 2 & 11 & 7.4 \\
\hline Benzoilecgonina + ac.11-nor- $\Delta^{9}$-thc-9 carboxílico & 3 & 1 & 4 & 2.7 \\
\hline Totales positivos & 31 & 4 & 35 & 23.6 \\
\hline No detectado & 85 & 28 & 113 & 76.4
\end{tabular}

Fuente: datos del estudio

Cuadro II

Resultados de los anÁlisis toxicológicos SEgún edAdes. Hospital Central de Valencia, Venezuela, 1995

\begin{tabular}{|c|c|c|c|c|c|}
\hline \multirow[b]{2}{*}{ Análisis } & \multicolumn{4}{|c|}{ Edad (años) } & \multirow{2}{*}{$\begin{array}{c}\text { Total } \\
(n=148)\end{array}$} \\
\hline & $<17(n=12)$ & $18-30(n=73)$ & $31-45(n=51)$ & $45-60(n=12)$ & \\
\hline Benzoilecgonina & 1 & 11 & 7 & 1 & 20 \\
\hline 11-nor- $\Delta^{9}$-thc-9 carboxílico & 1 & 6 & 2 & 2 & 11 \\
\hline Benzoilecgoninat 11-nor- $\Delta^{9}$-thc-9 carboxílico & 0 & 1 & 3 & 0 & 4 \\
\hline Total positivos & 2 & 18 & 12 & 3 & 35 \\
\hline No detectado & 10 & 55 & 39 & 9 & 113 \\
\hline
\end{tabular}

Fuente: datos del estudio

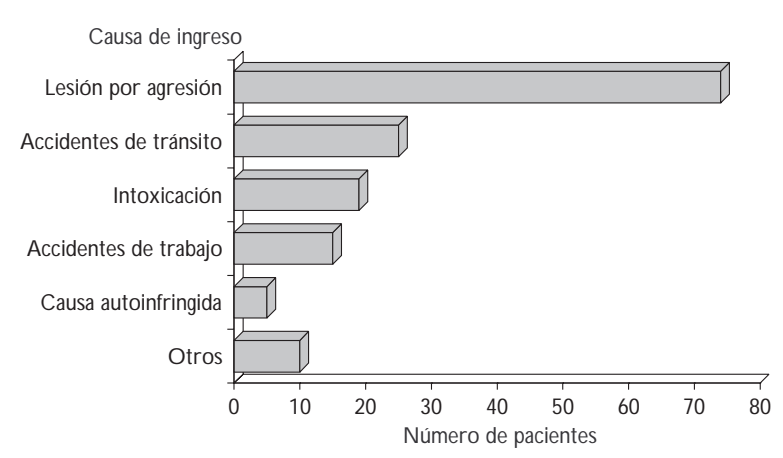

Fuente: datos del estudio

Figura 1. Distribución de los sujetos estudiados, según causa de ingreso. Hospital Central de VaLenCiA, Venezuela, 1995

solteros; 4 (11.4\%) vivían en unión libre, y 1 (2.9\%) era divorciado.

En cuanto al nivel educacional se encontró que 54 de los 148 individuos tenían educación primaria sola- mente; $53(35.8 \%)$ cursaban educación básica, y sólo $27(18.2 \%)$ se encontraban en cuarto y quinto año de educación secundaria. Entre los 35 pacientes positivos al consumo de drogas solas o asociadas 14 (40\%) habían aprobado la educación primaria; 17 (48.6\%) se encontraban cursando entre el séptimo y el noveno grado, y sólo 4 individuos (11.4\%) estaban en el cuarto y quinto año de educación secundaria.

Respecto a la actividad ocupacional, de los 148 pacientes $51(34.5 \%)$ eran albañiles; $42(28.4 \%)$ no refirieron qué actividad desempeñaban, y 14 (9.5\%) estaban desempleados. De los 35 casos que resultaron positivos a cualquiera de las drogas, $16(45.7 \%)$ eran albañiles y no tenían trabajo fijo.

\section{Discusión}

Los datos de este estudio revelan que el consumo de las drogas estudiadas, solas o asociadas, se hizo presente en $23.6 \%$ del total de los casos investigados; el consumo de cocaína fue el más alto, pues se observó en $57.14 \%$ del total de casos positivos (20/35) (cuadro I). Los mismos resultados obtuvo Mota, ${ }^{1}$ quien encon- 


\section{Cuadro III \\ Distribución de los sujetos estudiados según consumo de drogas y causa de ingreso. Hospital Central de Valencia Venezuela, 1995}

Causa de ingreso

ecgonina

Ac.11-nor- $\Delta^{9}$-thc9 carboxilico
Benzoil-ecgonina + ac.11-nor- $\Delta^{9}$-thc-9 carboxílico
Total $\quad \% *$

\begin{tabular}{|c|c|c|c|c|c|}
\hline Lesión por agresión ( $n=74)$ & 10 & 2 & 3 & 15 & 20.3 \\
\hline Causa autoinfringida $(n=5)$ & 2 & 0 & 0 & 2 & 40 \\
\hline Accidentes de tránsito $(n=25)$ & 0 & 4 & 0 & 4 & 16 \\
\hline Accidentes de trabajo $(n=15)$ & 1 & 3 & 1 & 5 & 33.3 \\
\hline Intoxicaciones $(n=19)$ & 4 & 1 & 0 & 5 & 26.3 \\
\hline $0 \operatorname{tros}(n=10)$ & 3 & 1 & 0 & 4 & 40 \\
\hline Total $(\mathrm{N}=148)$ & 20 & 11 & 4 & 35 & - \\
\hline
\end{tabular}

* Con base en positivos sobre $n$

Fuente: datos del estudio

tró que el consumo de cocaína era predominante en los 50 casos estudiados. Ambos resultados son consistentes con el trabajo de Salazar, ${ }^{2}$ quien menciona las principales drogas consumidas en Venezuela, donde la cocaína tiene el primer lugar.

En relación con el consumo según el sexo, no hubo diferencias significativas entre ambos parámetros. Esta situación se refleja de igual manera en estudios realizados en otros países: en un informe de la Secretaría de Salud de México y de los Centros de Integración Juvenil," se afirma que el fenómeno de la drogadicción en ese país se presenta en diferentes grupos de edades y en ambos sexos, pero que la población más afectada son los más jóvenes y del género masculino.

En el cuadro II se aprecia que la mitad de la muestra estuvo formada por individuos jóvenes (18-30 años), los cuales constituyen el grupo de la población más vulnerable a factores condicionantes del consumo de drogas descritos por De Franco, ${ }^{\ddagger}$ tales como: problemas de autoestima, ausencia de metas, curiosidad, búsqueda de placer, problemas familiares, falta de afecto, entre otros. Este rango de edades es comparable también con el estudio realizado por Hueso y colaboradores, ${ }^{16}$ en 100 pacientes farmacodependientes que consultaron el ambulatorio de San Bernardino, de la Fundación José Felix Ribas, Caracas, Venezuela; en esta

\footnotetext{
* Secretaría de Salud/Centros de Integración Juvenil. Epidemiología del abuso de drogas en la República mexicana: panorama comparativo con los Estados Unidos de América. México: Departamento de Información e Intercambio, SSA, 1992. Documento no publicado.

₹ De Franco S. Factores asociados al consumo de drogas. Caracas: Comisión Nacional Contra el Uso Ilícito de las Drogas, 1992. Documento no publicado.
}

investigación se encontró que la mayoría de los consumidores eran jóvenes varones menores de 26 años, con bajo nivel educacional, inestables laboralmente y con dificultades para mantener vínculos. En este estudio, del total de casos positivos al consumo de las drogas estudiadas, solas o asociadas, más de la mitad $(57.14 \%)$ correspondió a individuos con edades $\leq 30$ años. Sin embargo, al relacionar los resultados de laboratorio con grupos de edades $\leq 30$ años y $>30$ años no hubo asociación significativa entre ambos parámetros.

En la figura 1 se muestra que la causa de ingreso LA representó la mitad (74) del total de casos estudiados, proporción que resultó significativa $(p<0.05)$. El segundo lugar lo ocuparon los accidentes de tránsito cuyo origen y causa son variados y a menudo difíciles de establecer con exactitud; sin embargo, el factor humano es predominante en la mayoría de ellos, aparte de factores tales como condiciones de la vía y del vehículo.

Los hallazgos del cuadro III demuestran el estrecho vínculo que existe entre el consumo de drogas ilícitas y las causas de ingreso consideradas, lo que permite predecir que de cada cuatro ingresos a ese centro de salud, por causa traumática, al menos uno estuvo relacionado con el consumo de cocaína, marihuana o bazuco. El estudio de Lindenbaum y colaboradores, ${ }^{9}$ sobre ingresos por traumas a un centro de urgencias, reveló que $47.9 \%$ de los mismos estaban relacionados con crímenes violentos y que $74.5 \%$ resultó positivo a drogas ilícitas o de uso médico; asimismo, más de 50\% de los casos correspondió a consumo de cocaína. Por su parte, Sloan y colaboradores, ${ }^{17}$ en una investigación del mismo orden, encontraron $34 \%$ de consumo de marihuana y $37 \%$ de cocaína. Por otro 
lado, en un estudio de individuos que sufrieron accidentes de tránsito, Marzuk y colaboradore ${ }^{18}$ encontraron un consumo de cocaína del orden de $18.2 \%$.

Llama la atención que de los 25 ingresos por accidente de tránsito, sólo en cuatro se haya detectado consumo de marihuana. Es conocido el efecto de esta sustancia en cuanto a la alteración de la noción del tiempo, espacio y atención en los individuos consumidores. Soderstrom y colaboradores, ${ }^{19}$ del Centro Médico de la Universidad de Maryland, EUA, investigaron el probable consumo de marihuana y de otras drogas (alcohol, cocaína y fenciclidina) en conductores de automóviles y motocicletas que habían ingresado por accidentes de tránsito al Centro Médico. Los resultados revelaron que $2.7 \%$ de los conductores de automóviles y $32 \%$ de los conductores de motocicletas fueron positivos al ensayo del delta-9-THC. De igual forma, la relación entre los accidentes de tránsito y el consumo de drogas también fue un hallazgo importante del estudio de Del Río y Alvarez ${ }^{20}$ acerca de los patrones de consumo de drogas en conductores españoles, siendo la marihuana la droga de consumo más frecuente. Cabe destacar que si bien el alcohol es sumamente pernicioso para los conductores, el abuso de las drogas también los afectan en gran medida. ${ }^{21}$

Respecto a la combinación de cocaína y alcohol, de los 20 casos que resultaron positivos al consumo de ésta y/o de bazuco, la mitad se asoció con el consumo de alcohol; entre los cuatro sujetos positivos a cocaína, bazuco y/o marihuana, un caso estuvo asociado con la ingesta de alcohol, la cual fue determinada por observación del estado en que ingresaba el paciente y por el cuestionario aplicado. Al relacionar causa de ingreso y resultado de laboratorio no se obtuvo una asociación significativa desde el punto de vista estadístico.

En lo que concierne al nivel educativo, existe una estrecha vinculación entre éste, la marginalidad social y el consumo de drogas, por lo que estos resultados se asemejan a los notificados por Miguez, ${ }^{22}$ en San José de Costa Rica, sobre la situación de marginalidad social y el consumo de sustancias como marihuana, fármacos, disolventes industriales, etcétera.

Respecto a la actividad ocupacional se observó un marcado índice de inestabilidad laboral, lo cual refleja la posibilidad de una mayor tendencia al consumo de drogas en aquellos individuos que presentan inestabilidad laboral y de pareja. En los casos positivos a los ensayos de cocaína y marihuana, se encontró que la ocupación prevaleciente fue la de albañil u otras ocupaciones similares relacionadas con el área de la construcción.

Los resultados del presente estudio demuestran que existe una relación entre el consumo de drogas y el ingreso de pacientes al servicio de emergencias del Hospital Central de Valencia, Venezuela, por causas traumáticas. La prevalencia entre los parámetros estudiados fue de $23.6 \%$, con una mayor tendencia al consumo de drogas en los hombres que en las mujeres, lo cual confirma los hallazgos publicados al respecto. ${ }^{18}$ Se encontró que la droga más consumida es la cocaína, lo que corrobora que es la droga de elección de los consumidores.

La afectación del grupo de edad de entre 18 y 30 años denota un problema de salud pública importante pues recae sobre un sector de la población en edad productiva, ocasionando daño en la salud integral de la persona, reducción de la calidad de vida y de la capacidad productiva, y comprometiendo el bienestar del grupo familiar del accidentado y de la sociedad.

Es necesario que los organismos competentes lleven a cabo el diseño y la puesta en marcha de políticas dirigidas a difundir información acerca del consumo de drogas, sus efectos nocivos en la salud, las formas de prevención y las posibilidades de rehabilitación y reinserción social.

El presente estudio constituye una etapa preliminar para futuras investigaciones sobre el abuso de drogas en población traumatizada. Por este motivo, se recomienda llevar a cabo estudios sobre sujetos que ingresen a los servicios de urgencias por lesiones traumáticas, realizando análisis toxicológicos rutinarios para detectar el consumo de drogas, además de la ingestión de alcohol. De esa manera, podría contarse con una proyección del problema, teniendo en cuenta que la población más comprometida es la estudiantil, joven y trabajadora, esto es, el futuro de Venezuela.

Cabe subrayar, además, la importancia de que en los centros de urgencias se lleven a cabo rutinariamente los análisis toxicológicos mencionados en el momento del ingreso del paciente traumatizado, y que se anexen los resultados en las fichas de ingreso, garantizando así un mejor registro de esos casos.

Por otra parte, los estudios específicos donde se analicen a fondo los efectos de la interacción de las drogas aquí mencionadas, también pueden hacer una valiosa aportación a la presente investigación.

\section{Agradecimientos}

Las autoras agradecen al Consejo de Desarrollo Científico Humanístico y Tecnológico (CODECIHT) la subvención otorgada a esta investigación; asimismo, a las licenciadas Maritza Salazar y Sonia Barría por permitir la realización de este trabajo junto con el programa Vigilancia Epidemiológica del Consumo de Drogas 1995; a la Dirección del Hospital Central de Valencia y 
al Area de Emergencias, e igualmente al personal médico y de enfermería, que hicieron posible la recolección de la información y la toma de las muestras.

\section{Referencias}

1. Mota G. U sos de anestésicos generales en consumidores de cocaína y marihuana (tesis). Caracas: Universidad Central de Venezuela, 1993.

2. Salazar M, Ramos G. Diagnóstico epidemiológico del consumo de sustancias psicoactivas en una universidad venezolana. Valencia, Venezuela: Artelit, 1994.

3. González A. Criterios toxicológicos del abuso de drogas. I Congreso Internacional de Educación Superior en la Problemática de la D roga; 1991 nov 26-29; Valencia, Venezuela.

4. Tardiff $K$ y Gross E. An analysis of cocaine positive fatalities. JFSCA 1989;34:53-63.

5. Bailey D, Shaw R. Cocaine and methamphetamine related deaths in San Diego County (1987): Homicides and accidental overdoses. JFSCA 1989:34:407-422.

6. Castro M, García G, Rojas E, Serna J. Conducta antisocial y uso de drogas en una muestra nacional de estudiantes mexicanos. Salud Publica Mex 1988;30:216-226.

7. Spunt $B$, Goldstein P, Brawnstein H, Fendrich M.The role of marijuana in homicides. Int J Addict 1994;29:195-213.

8. Parran T Jr, Tase J,Anderson B,A delman C. Mandatory toxicology testing and chemical dependence consultation follow-up in a level-one trauma center. J Trauma 1995;38:278-280.

9. Linbenbaum G, C arrol S, D askal Y. Patterns of alcohol and drug abuse in an urban trauma center:The increasing role of cocaine use. J Trauma 1989;29:1654-1658.
10. Alcaldía de Medellín/Programa de las $\mathrm{N}$ aciones Unidas para la Fiscalización Internacional de Drogas/Dirección Nacional de Estupefacientes/ Plan Municipal de Prevención en Medellín. Sistema de vigilancia epidemiológica sobre el uso indebido de sustancias psicoactivas (VESPA). Medellín, Colombia: Marín Vieco, 1993.

11. Salazar M. Epidemiología del consumo de sustancias psicoactivas.Valencia, Venezuela: Universidad de Carabobo, 1996.

12. 0 ellerich M. Enzymeimmunoassays in clinical chemistry: Present status and trends. J Clin Chem Biochem 1980;18:197-208.

13. Frings $C$, Battaglia D,W hite R. Status of drugs of abuse testing in urine underblind conditions:An AACC study. Clin Chem 1989;35(5):891-894. 14. System 0 perator's Manual. San José (CA): Syva ETS, 1992.

15. National Institute on Drug Abuse. Urine testing for drugs of abuse. Research Monograph 73. NIDA, 1976.

16. Hueso $\mathrm{H}$, Lara E, Cuervo F. Estudio exploratorio de 100 pacientes farmacodependientes, patrones de consumo, multicausalidad, personalidad y otros diagnósticos asociados. Arch Venez Psiquiatr N eurol 1991; 37:15-20

17. Sloan E, Zalenski R, Smith R. Toxicology screening in urban trauma patients: Drug prevalence and its relationship to trauma severity and management. J Trauma 1989;29: 1647-1653.

18. Marzuk P, Kenneth T, Leon A, Stajic M, Morgan E, Mann J. Prevalence of recent cocaine use among motor vehicle fatalities in $\mathrm{N}$ ew York City. JAMA 1990;263:250-256.

19. Soderstrom C, D ischinger P, Kerns T, Trifillis A. Marijuana and other drug use among automobile and motorcycle drivers treated at a trauma center. Accid A nal Prev 1995;27:131-135.

20. Del Río M, Alvarez J. Illegal drug taking and driving: Patterns of drug taking among Spanish drivers. D rug Alcohol D epend 1995;37:83-86.

21. O rsay E, Doan L, Lewis $R$, Lucke $R$, Ramakrishnan V. The impaired driver: Hospital and police detection of alcohol and other drugs of abuse in motor vehicle crashes. Ann Emerg Med 1994;24:51-55.

22. Miguez H. Farmacodependencia de la pobreza. Su prevalencia en C osta Rica. Acta Psiquiatr Psicol Am Lat 1984;30:255-263. 